two national sexual behavioural surveys among Indigenous youth conducted in Australia and New Zealand, as well as summarise the evidence, the gaps and challenges with a social determinants lens. We will discuss opportunities and explore innovative ideas that may address these disparities via an interactive panel and group discussions.

\section{S12.1 SEXUAL HEALTH OF MAORI ADOLESCENTS, FINDINGS FROM THE YOUTH'12 NATIONAL YOUTH HEALTH AND WELLBEING SURVEY IN SECONDARY SCHOOLS THROUGHOUT NEW ZEALAND}

Terryann Clark*. University of Auckland, Auckland, New Zealand

\subsection{6/sextrans-2015-052270.60}

Aim To describe the prevalence and trends of sexual health behaviours for Māori youth in New Zealand.

Method The Youth 2000 survey series are anonymous, representative, cross-sectional, self-administered surveys undertaken in 2001, 2007 and 2012 with over 27,000 New Zealand secondary school students aged $12-18$ years.

Results Data from the surveys highlight that in 2012, 36\% of Māori had ever had sex. This was significantly lower than in previous waves of the survey (47.6\% in 2001, 55.8\% in 2007). Similarly those who report being currently sexually active has declined over time $33.5 \%$ in $2001,41.8 \%$ in 2007 and $26.5 \%$ in 2012). Despite the trends to fewer Māori youth engaging in sexual behaviour in secondary school, the use of contraception (51.3\% in $2001,52.2 \%$ in 2007 and $48.4 \%$ in 2012) and condoms (46.4\% in 2001, 36.1\% in 2007 and 43.8\% in 2012) has not improved. Compared to New Zealand European (NZE) students, Māori are significantly more likely to be sexually active (OR 1.50, 95\% CI 1.24, 1.83) and less likely to use contraception (OR 0.50, 95\% CI 0.38, 0.66). There was no difference in condom use between Māori and NZE. Māori also reported that they were less able to access primary care (GP) they required in the previous 12 months (OR $0.84,95 \%$ CI $0.72,0.99$ ).

Conclusion Māori youth in New Zealand are delaying sexual activity, but those who are sexually active are particularly vulnerable to STIs. Specific strategies are required that improve access to appropriate contraception and condom use for Māori.

\section{S13 - Microbiome of the genital tract, host immune responses and STI interactions}

\section{S13.1 THE MANY FACETS OF THE VAGINAL MICROBIOME IN HEALTH AND DISEASE}

Jacques Ravel*. University of Maryland School of Medicine, Baltimore, USA

\subsection{6/sextrans-2015-052270.61}

The vaginal microbiota forms the first line of defense against sexually transmitted infection (STIs). Population-based surveys of the bacteria inhabiting the vagina have shown that several kinds of vaginal microbiota exist, that differs in bacterial composition and abundance. Further, in some women, these communities are dynamic and can change over short period of time, while in other, they are highly stable and do not change. The impact of both composition and dynamic of the vaginal microbiota on the susceptibility to diseases is still poorly understood. The application of modern genomic technologies, ecological principles and in vitro modelling affords a better understanding of the role of vaginal microbiome in health and diseases. Metagenomic sequencing provides a comprehensive view of the genetic make up of the bacterial species comprising the vaginal microbiome, and highlights associations between different genomic species of the same genus, or strains of the same species and their contribution to the protective properties of the vaginal microbiome. For example, the genomic make up of certain vaginal bacteria correlates with their ability to maintain a stable and protective vaginal microbiome. Further, the use of in vitro three-dimensional models of cervical epithelial cell lines is a good surrogate to evaluate the contribution of microbial products to the function of the vaginal microbiome. Using a 3D model of cervical epithelial cells, we have shown that the production of different isomers of lactic acid by Lactobacillus spp. is associated with protection against chlamydial infection in a $\mathrm{pH}$ dependent manner. Understanding the vaginal microbiome structure and functions is critical to devise novel and personalised strategies to maximise women's health.

\section{S13.2 GENITAL IMMUNOLOGY, THE MICROBIOTA AND HIV TRANSMISSION}

Rupert Kaul*. University of Toronto, Toronto, Canada

\subsection{6/sextrans-2015-052270.62}

Background The probability of HIV acquisition after a sexual exposure is low, but surprisingly heterogeneous. Key mucosal immune determinants of susceptibility include genital epithelial disruption and/or the presence of activated $\mathrm{CD}^{+} \mathrm{T}$ cell in the genital mucosa, both of which have been linked to mucosal inflammation. We hypothesise that the genital microbiota may alter mucosal immunology and hence HIV susceptibility.

Methods We have carried out ex vivo studies of cervical, foreskin and semen mucosal immunology and in vivo studies of HIV acquisition in participant cohorts from Canada and East Africa. Studies use endocervical cytobrush samples and ectocervical biopsies from female participants, as well as foreskin tissues obtained during elective penile circumcision and semen samples from male participants. Mucosal T cell parameters and soluble genital immune factors associated with HIV transmission are assessed, co-infection diagnostics performed and the bacterial microflora assayed using $16 \mathrm{~S}$ rRNA gene-based pyrosequencing and quantitative PCR.

Results In HIV-uninfected individuals, asymptomatic herpes infection was characterised by mucosal $\mathrm{T}$ cell immune activation and increased CD4 expression of the HIV co-receptor CCR5 and/or a4b7, in the absence of any local elevation in pro-inflammatory cytokines. However, while a vaginal microbiome characterised by bacterial diversity was not associated with mucosal cellular alterations, microbiota alterations in both the vagina and foreskin were associated with elevated pro-inflammatory cytokines, and the latter with a proteomic profile suggestive of epithelial disruption. In HIV-infected men, the semen microbiome was altered and linked to levels of HIV RNA in the seminal plasma, as well as to local levels of pro-inflammatory cytokines. Conclusion The microbiota of the vagina and foreskin are associated with HIV susceptibility, and with important alterations in mucosal immunology that appear to be quite distinct to those induced by the HIV-enhancing co-infection HSV-2. This may lead to new clinical HIV prevention strategies. 